\title{
Prevalencia de diabetes mellitus gestacional en una población de Medellín de 1999 - 2000: valor predictivo positivo de la prueba tamiz y comparación de los criterios de la NDDG y la ADA
}

\author{
Hernán Cortés Y.*, Iván Ocampo T. *, Alberto Villegas P.**
}

\section{RESUMEN}

INTRODUCCION: En 1997 la Asociación Americana de Diabetes (ADA), recomendó cambiar los criterios de tamización y diagnóstico para la diabetes gestacional, no tamizando a las pacientes de bajo riesgo y adoptando para el diagnóstico los criterios de Carpenter y Coustan.

En nuestro medio no conocemos la incidencia de la diabetes, ni los niveles de riesgo.

MÉTODOS: Se realizó una descripción prospectiva de pacientes gestantes que realizaron control prenatal de agosto de 1999 a julio de 2000, en el CAA central del ISS de la ciudad de Medellín; a todas estas se les realizó la prueba tamiz (prueba de O'Sullivan) entre las semanas 24-28 de la gestación y la prueba oral de tolerancia a las que lo requirieron.

Se realizó una encuesta telefónica luego del parto para obtener los datos del resultado materno y neonatal.

RESULTADOS: Se evaluaron 1726 pacientes de manera consecutiva, encontrando una prevalencia de diabetes gestacional de $1.43 \%$, la cual aumentó a $2.03 \%$ cuando se aplicaron los nuevos criterios.

Se determinó el valor predictivo positivo de la prueba tamiz, demostrando que no es diagnóstica con ningún valor.

En cuanto al resultado neonatal el 20.5\% de los bebés presentaron macrosomía, 13\% en el grupo que cumplió los criterios de la NDDG y de $40 \%$ en los que cumplieron los de la ADA.

CONCLUSIONES: En este grupo de pacientes se encontró una prevalencia baja de diabetes gestacional en comparación a poblaciones de otras regiones, no se logró demostrar que el adoptar los nuevos criterios mejore el pronóstico materno y neonatal; se deben realizar nuevos estudios que permitan una mejor selección de la población que se va a someter a la prueba tamiz para optimizar los recursos.

PALABRAS CLAVE: Diabetes mellitus gestacional, incidencia, prueba tamiz, valor predictivo positivo, macrosomía, diagnóstico. 


\section{SUMMARY}

BACKGROUND: In 1997 the American Diabetes Association (ADA), recommended a change in the screening and diagnosis criteria: no screening for low risk patients and adoption of the diagnosis criteria by Carpenter and Coustan.

We do not know the incidence in our population or whether we are or not a high-risk group for diabetes.

METHODS: A descriptive series was carried out from August 1999 to July 2000. Pregnant women who had prenatal checkups in the central CAA of Medellín's SSI; they underwent to screening with the O'Sullivan test between 24 - 28 gestational weeks, and the tolerance oral test for those who needed it.

A phone survey was done after birth for getting the maternal and neonatal results.

RESULTS: 1726 patients were evaluated. An incidence of $1.43 \%$ was found and increased to $2.03 \%$ when the new criteria were applied.

The positive predictive value of the screening test was determined, demonstrating that it was not diagnostic with any value.

20.5\% of infants presented macrosomia. $13 \%$ in the NDDG criterion group and $40 \%$ in the ADA criterion group.

CONCLUSION: In this group of patients, a low incidence of gestational diabetes was found compared with other population, we did not achieve to demonstrate that adopting the new criteria improved the maternal and neonatal prognosis; it should be carried out new surveys which permit a better population selection who are going to undergo the screening test for optimizing resources.

KEY WORDS: Gestational diabetes mellitus, incidence, screening test, positive predictive value, macrosomia, diagnosis.

\section{INTRODUCCIÓN}

A partir de 1997 la cuarta conferencia internacional del grupo de trabajo en diabetes mellitus gestacional, recomendó cambiar los criterios de tamización y de diagnóstico. Para la tamización sugieren no realizarla de manera universal (a todas las embarazadas), sino según los factores de riesgo de cada paciente, aduciendo que en las pacientes de bajo riesgo no es costo-beneficioso realizarla debido a su baja incidencia ${ }^{1}$.

El grupo de bajo riesgo estaría conformado por mujeres menores de 25 años, de peso normal (IMC < 25), sin antecedentes familiares de diabetes y que no pertenecen a grupos étnicos de alto riesgo, a saber: latinos, negros, indígenas americanos y australianos, entre otros; es de anotar que el grupo de latinos al que ellos se refieren probablemente es al de los inmigrantes, pero esto no queda claro.
Según esto nuestra población (latinos) podría pertenecer al grupo de alto riesgo, con una mayor incidencia de Diabetes Mellitus (DM) y Diabetes Mellitus Gestacional (DMG), sin embargo en nuestro medio no existen estudios de la incidencia de estas patologías.

En cuanto a los criterios diagnósticos, la cuarta conferencia recomienda disminuir los valores de la National Diabetes Data Group (NDDG) y adoptar los criterios propuestos por Carpenter y Coustan (Tabla 1). Sin embargo esta recomendación ha generado controversia y algunos estudios han reportado un aumento en la incidencia de DMG y en los costos, sin encontrar mejoría en el resultado materno y neonatal ${ }^{2}$.

Existe en nuestro medio la idea de que valores en la prueba tamiz $>180 \mathrm{mg} / \mathrm{dl}$ serían diagnóstico de DMG y que en estos casos no se requiere la prueba confirmatoria (3); sin embargo, ésto no ha sido avalado ni es 
recomendado por los grupos de trabajo en DMG.

Los objetivos de este trabajo son: determinar la prevalencia de DMG en un grupo de mujeres embarazadas en nuestro medio, determinar si el valor predictivo positivo de la prueba tamiz y comparar los criterios de la NDDG con los de Carpenter y Coustan en cuanto al resultado materno y neonatal.

Tabla 1

Criterios diagnósticos de diabetes mellitus gestacional

\begin{tabular}{|lcc|}
\hline & NDDG & CARPENTER-COUSTAN \\
\hline Ayunas & 105 & 95 \\
1 hora & 190 & 180 \\
2 horas & 165 & 155 \\
3 horas & 145 & 140 \\
\hline
\end{tabular}

\section{MATERIALES Y MÉTODOS}

Se realizó una descripción prospectiva del $1^{\circ}$ de agosto de 1999 al 31 de julio de 2000, en las embarazadas que asistieron a control natal al CAA central del Instituto de Seguro Social (ISS) de la ciudad de Medellín.

A todas las embarazadas sin diagnóstico previo de DM se les realizó la prueba de tamización entre las semanas 24 a 28 de gestación con 50 gramos de glucosa, a las pacientes con valores mayores o iguales a 135 $\mathrm{mg} / \mathrm{dl}$ a la hora en este examen, se les realizó en ayunas la prueba oral de tolerancia a la glucosa (POTG) con 100 gramos, el resto salieron del estudio (sólo se tomaron para calcular la prevalencia).

Las pacientes con prueba de tamiz positiva se dividieron en tres grupos, el primero fue conformado por las pacientes que llenaron los criterios de la NDDG luego de la POTG con 100 grs, el segundo por las que sólo alcanzaron los nuevos criterios de la ADA con la misma prueba y por último, las pacientes con PTOG normal (sanas, no diabéticas). Para esta época aún no se aceptaban los criterios de Carpenter y Coustan en esta institución, por lo tanto sólo a las pacientes del primer grupo se les hacía diagnóstico de DMG y se remitían al servicio de alto riesgo obstétrico para el manejo y control de su patología; las pacientes que no lograban el control de la glicemia con dieta (glicemia en ayunas < $105 \mathrm{mg} / \mathrm{dl}$ y dos horas posprandial < $120 \mathrm{mg} / \mathrm{dl}$ ) se les iniciaba insulina, los otros controles y manejo dependían de cada paciente y su médico tratante; las pacientes de los grupos 2 y 3 continuaban su control prenatal en el CAA central con el médico general y la enfermera.
La glucosa plasmática fue medida por le método de glucosa oxidasa y se realizó por el personal del laboratorio del CAA central, los valores alterados en la prueba tamiz en la POTG con 100 grs fueron confirmados.

Las pacientes de los grupos 1 y 2 eran llamadas a sus hogares al terminar el embarazo y se les realizaba una encuesta que incluía el peso, talla, antecedentes familiares de DM, antecedentes obstétricos, patologías durante el embarazo, vía del parto, edad gestacional a la terminación del embarazo, datos del bebé como peso, talla y patologías, tratamiento recibido (dieta o insulina) y el resultado de la POTG con 75 grs a las seis semanas posparto; se calculó el índice de masa corporal (IMC), con la ecuación peso/talla ${ }^{2}$.

\section{ANÁLISIS ESTADÍSTICO}

Se estableció el porcentaje de predicción positivo de la prueba tamiz a diferentes puntos de corte, o sea que porcentaje de pacientes con prueba tamiz positiva se diagnosticaron como diabéticas con la POTG; se tomaron como macrosómicos los bebés con un peso mayor de 4000 grs y como grandes para la edad gestacional aquellos con un peso mayor al percentil 95 para la edad.

Para los datos demográficos de ambos grupos se analizaron las variables cuantitativas de edad e IMC para constatar si provenían de poblaciones de distribución normal utilizando la prueba de Shapiro-Wilk. Para la comparación de los dos grupos se utilizó la prueba $\mathrm{t}$ para diferencias de medias con varianza desigual o en su defecto la prueba $U$ de Mann-Whitney; para comparar la variable antecedentes familiares se utilizó el chi cuadrado de homogeneidad.

Los resultados se presentaron por medio de tablas y gráficos.

\section{RESULTADOS}

En total se evaluaron 1726 mujeres embarazadas de manera consecutiva sin antecedente de DM, a las que se les realizó la prueba tamiz con 50 grs de glucosa, en 1423 pacientes la prueba fue negativa (glicemia $<135$ $\mathrm{mg} / \mathrm{dl})$ y en 303 pacientes fue positiva (17.5\% de la población).

De estas 303 pacientes, 58 (19.1\%) no se realizaron la POTG con 100 grs, (no se incluyeron en los datos) quedando 245 que si se sometieron a ella, las cuales se distribuyeron de la siguiente manera (Tabla 2):

De estas, 24 pacientes (1.43\%) cumplieron los criterios de la NDDG y 34 pacientes 
(2.03\%) los de Carpenter y Coustan, con un aumento en la incidencia del $70.4 \%$ cuando se aplicaron estos últimos.

Tabla 2

Distribución de la prueba tamiz

\begin{tabular}{|lcc|}
\hline $\begin{array}{l}\text { GLICEMIA } \\
\text { (mg/d) }\end{array}$ & $\begin{array}{c}\text { NÚMERO DE } \\
\text { PACIENTES }\end{array}$ & $\begin{array}{c}\text { PORCENTAJE } \\
\mathbf{( \% )}\end{array}$ \\
\hline $135-139$ & 43 & 17.5 \\
$140-150$ & 73 & 29.8 \\
$151-160$ & 50 & 20.4 \\
$161-170$ & 43 & 17.5 \\
$171-180$ & 13 & 5.3 \\
$181-190$ & 10 & 4.08 \\
$191-200$ & 7 & 2.8 \\
$>200$ & 6 & 2.45 \\
Total & 245 & 100 \\
\hline
\end{tabular}

La edad promedio de las pacientes fue de 33.6 años (20 - 44 años), 3 eran menores de 25 años; el IMC fue de 24.1 (31.2 - 18.5), 12 pacientes (35.2\%) tuvieron un IMC mayor a 25 y 23 mujeres no tenían antecedentes familiares de diabetes. (Sin diferencia estadística entre los grupos) Tabla 3.

Tabla 3

Datos demográficos

\begin{tabular}{|lccc|}
\hline & $\begin{array}{c}\text { Promedio } \\
\text { general }\end{array}$ & NDDG & ADA \\
\hline Edad (años) & $33(20-44)$ & 33.4 & 34.2 \\
MMC $^{2}$ & $24.1(18.5-31.2)$ & 24.4 & 23.2 \\
Antec. Familiar $^{3}$ & 12 & 8 & 4 \\
\hline
\end{tabular}

${ }^{1}$ Prueba $t$ diferencia de medias, $t=-0.191, p=0.806$.

${ }^{2}$ Prueba U de Mann-Whitney, $U=78.5, p=0,542$

${ }^{3}$ Prueba chi-cuadrado, $x 2=0,992, p=0.319$

El gráfico 1 muestra la distribución de las pacientes según el valor en la prueba tamiz, encontrando que entre 135 - $139 \mathrm{mg} / \mathrm{dl}$ el valor predictivo positivo de esta prueba fue de cero, con valores > a $180 \mathrm{mg} / \mathrm{dl}$ fue de $41.1 \%$ y aún con valores mayores a $200 \mathrm{mg} / \mathrm{dl}$ no es del 100\% (sólo de 50\%).

Valor predictivo positivo de la prueba tamiz (porcentaje de pacientes diagnosticadas como diabéticas con la POTG de 100 grs.)

Se diagnosticó DMG en 3 pacientes que se considerarían de bajo riesgo (menores de 25 años, con IMC $<25$ y sin historia familiar de diabetes).

En cuanto al resultado materno y neonatal, entre las 24 pacientes que cumplieron los criterios de la NDDG, se presentaron 3 casos de bebés con macrosomía (13\%) (peso > 4000 grs), 7 pacientes padecieron preeclampsia (29\%) y a 13 (56\%) se les realizó cesárea. De una paciente no se obtuvieron datos.

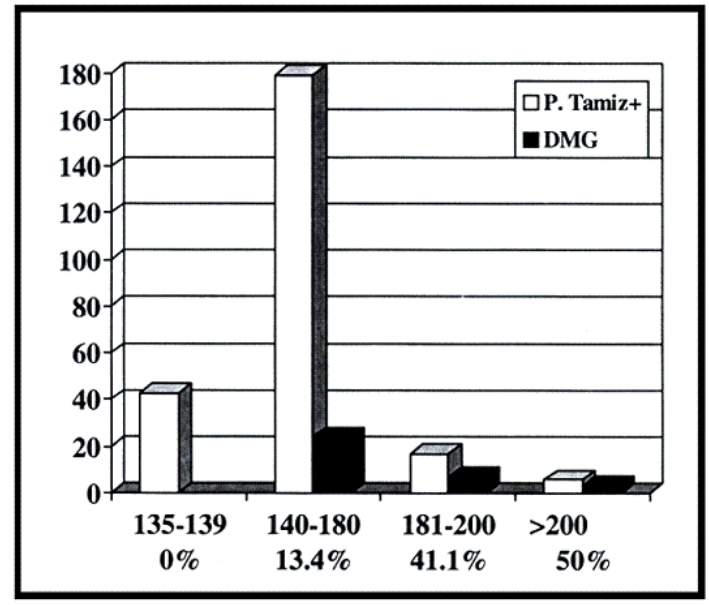

Gráfico $002 \mathrm{i} 01$

Valor predictivo positivo de la prueba tamiz (porcentaje de pacientes diagnosticadas como diabéticas con la POTG de 100 grs.)

De las 10 pacientes que sólo alcanzaron los criterios de Carpenter y Coustan, de 2 no se obtuvieron datos, se presentaron 3 casos de macrosomía fetal (30\%) y un bebé grande para la edad gestacional de 34 semanas con un peso de 3600 grs (percentil > 95 para la edad), que nació por cesárea por preeclampsia en la madre; dos casos de preeclampsia (20\%) y cuatro embarazos que se terminaron por cesárea (40\%).

En total el número de bebés con macrosomía fue de 7 (20.5\%).

\section{CONCLUSIONES}

En este trabajo se encontró que la prevalencia de DMG en este grupo de pacientes fue de $1.43 \%$, la cual es muy baja si se compara con la reportada en otras poblaciones, donde se alcanzan valores de $15 \%$ como en los indígenas australianos, $14.3 \%$ en los indios Zuni y 4\% en la población general de E.U, entre otras y se compara a la de las poblaciones de bajo riesgo, como las Norte Americanas blancas no hispánicas (1.6\%), las mujeres de Inglaterra (1.2\%) y las Chinas de Taiwán $(0.6 \%)^{4}$.

Cuando se usaron los criterios de Carpenter y Coustan la prevalencia pasó a $2.03 \%$ con un aumento del $70.4 \%$, la cual sigue siendo baja.

Cabe recordar que el comité de expertos de la cuarta conferencia en DMG, consideró a los 
latinos como un grupo de alto riesgo para la DMG; sin embargo, los hallazgos de este estudio (baja prevalencia) y otros realizados en México y Chile donde se encontró una incidencia del 6 y el $0 \%$ respectivamente ${ }^{4-5}$, parecen demostrar que hay diferencias entre regiones y con el latino que vive en Norte América con respecto al que no ha emigrado, tal vez por cambios en los hábitos alimenticios; por lo tanto, cada país y región debe conocer su propia incidencia.

En Cali (Colombia) durante los años 1989 - 1996, se encontró una incidencia de DMG de $0.34 \%$, lo que refuerza la idea que somos un país de bajo riesgo ${ }^{9}$.

En la institución donde se realizó este trabajo se considera como positiva la prueba tamiz cuando la glucemia a la hora es mayor o igual a $135 \mathrm{mg} / \mathrm{dl}$, ocurriendo ésto en el 17.5\% de la población, lo cual se reduciría a $15 \%$ si se toma el valor recomendado por la American Diabetes Association (ADA) de $140 \mathrm{mg} / \mathrm{dl}$, además en este grupo no se diagnosticó DMG en ninguna paciente con valores en la prueba tamiz entre 135 - $139 \mathrm{mg} / \mathrm{dl}$, por lo que se pudiera obviar la POTG de 100 grs en estos casos, con disminución en los costos.

Aunque el uso de un valor alto en la prueba tamiz no se reconoce como suficiente para diagnosticar DMG (obviando la POTG de 100 grs), algunos autores recomiendan clasificar como diabéticas a las pacientes con valores $>180 \mathrm{mg} / \mathrm{dl}$ en la prueba tamiz ${ }^{3,6}$; $\sin$ embargo, en este estudio y otro ${ }^{7}$ se demuestra que aún con valores $>200 \mathrm{mg} / \mathrm{dl}$ en la prueba tamiz, no se hace diagnóstico en el $100 \%$ de los casos cuando se aplica la POTG con 100 grs.

Debido a la baja incidencia de DMG en nuestra población quedaron muy pocas pacientes diagnosticadas como diabéticas en cada grupo por lo que no se pueden hacer comparaciones de significancia estadística.

La discusión continúa abierta aunque el cambio en los criterios es lo recomendado por la ADA, algunos estudios han encontrado que adoptar estos nuevos criterios llevaría a un gran aumento en la incidencia (54\%) y en los costos, afectando sólo de manera mínima la prevalencia de bebés con macrosomía (disminuyendo de 20 a 19.8\%) ${ }^{2}$, y otros han encontrado que no realizar la prueba tamiz en grupos de bajo riesgo dejaría sin diagnóstico a un número importante de pacientes (2.8\% de la población) las cuales estarían expuestas a la morbilidad de esta patología (bebés con macrosomía, cesáreas, uso de insulina, etc.) ${ }^{8}$.
En este estudio en tres pacientes de bajo riesgo se diagnosticó DMG $(8.8 \%$ de las diabéticas), que se hubieran quedado sin diagnóstico si se adoptan los criterios de la ADA (tamización selectiva).

Se deben realizar otros estudios encaminados a evaluar otros factores de riesgo $o$ indicadores que permitan una mejor selección de la población que se va a someter a la prueba tamiz para optimizar los recursos y que aclaren si el cambio en los criterios mejora el resultado materno y neonatal.

\section{AGRADECIMIENTOS}

$\mathrm{Al}$ director del CAA central del ISS de la ciudad de Medellín, Doctor León Jairo Montaño.

Al personal del Laboratorio del CAA y principalmente a la Bacterióloga Ana Zuluaga y a Víctor Hugo Bernal.

A Daniel Camilo Aguirre A. estadístico informático, por la asesoría en estadística.

$\mathrm{Al}$ centro de investigaciones médicas (CODI) de la Facultad de Medicina de la Universidad de Antioquia.

\section{BIBLIOGRAFÍA}

1. Metzger BE, Coustan DM, Organizing Committee. Summary and recommendations of the fourth international workshop conference on Gestational Diabetes Mellitus. Diabetes Care 1998;21 Suppl 2:B1617.

2. Schwartz ML, Ray WN, Lubarsky SL. The diagnosis and classification of gestational diabetes mellitus: Is it time to change our tune? Am J Obstet Gynecol 1998;180:1560-71.

3. Jubiz A. Diabetes. En: Botero J, Jubiz A, Henao G. Obstetricia y Ginecología. Texto integrado. 5a. Ed. 1994. p. $213-18$.

4. King H. Epidemiology of glucose intolerance and gestational diabetes in women of childbearing age. Diabetes Care 1998; 21 Suppl 2:B9-13.

5. Tamez HE, Rodríguez M, Trevino M, et al. Experience with a screening program for gestational diabetes. Ref Invest Clin. 1993;45:453-6.

6. Carpenter M, Coustan DM. Criteria for screening tests for gestational diabetes. Am J Obstet Gynecol 1982;144:768.

7. Sacks DA, Abu-Fadil S, Karten G, et al. Screening for gestational diabetes with the one-hour 50 -g glucose test. Obstet Gynecol 1987;70:89.

8. Moses R, Moses J, David W. Gestational Diabetes: Do lean young Caucasian women need to be tested? Diabetes Care 1998;21:1803-6.

9. Gómez G, Mesa JC. Diabetes y Embarazo. Evaluación del programa en la consulta de endocrinología del Hospital Universitario del Valle 1989 - 1996. Rev Colomb Obstet Ginecol 1997;48(4):239-41. 